\title{
Philosophiques
}

\section{Les « objections " au cours Éthique et culture religieuse : retour sur les enjeux du débat}

\section{Stéphanie Tremblay}

Volume 43, numéro 2, automne 2016

URI : https://id.erudit.org/iderudit/1038219ar

DOI : https://doi.org/10.7202/1038219ar

Aller au sommaire du numéro

Éditeur(s)

Société de philosophie du Québec

ISSN

0316-2923 (imprimé)

1492-1391 (numérique)

Découvrir la revue

Citer ce document

Tremblay, S. (2016). Les « objections » au cours Éthique et culture religieuse : retour sur les enjeux du débat. Philosophiques, 43(2), 499-507.

https://doi.org/10.7202/1038219ar d'utilisation que vous pouvez consulter en ligne.

https://apropos.erudit.org/fr/usagers/politique-dutilisation/ 


\title{
Les «objections » au cours Éthique et culture religieuse: retour sur les enjeux du débat
}

\author{
STÉPHANIE TREMBLAY \\ Département de sciences des religions, UQAM \\ tremblay.stephanie.2@uqam.ca
}

\section{Introduction}

Dans son premier chapitre, Éduquer au pluralisme: le débat, Georges Leroux retrace les différents types d'opposition frontale au pluralisme en éducation à travers les trois objections emblématiques adressées au programme Éthique et culture religieuse (ECR). Ces trois courants critiques représentent des postures assez différentes, de par les acteurs qui les portent et leurs préoccupations politiques, éducatives et philosophiques, mais qui convergent en même temps sur d'autres aspects, notamment dans leur nostalgie d'une forme plus homogène de la «culture publique commune» ou de la «québécitude». Nous proposons ici de revenir brièvement sur chacune de ces objections en mettant l'accent sur les convergences sociologiques de ces trois discours, eu égard à la conception normative des relations entre religion et politique, et du lien social. Après avoir défini l'horizon commun de ces critiques, nous nous pencherons plus spécifiquement sur les objections catholiques à la formation en "culture religieuse", qui à notre sens, s'inscrivent dans deux logiques argumentaires fondamentalement différentes, dont les rapports au pluralisme ne peuvent être confondus.

\section{Trois objections, un horizon commun}

Pour Leroux, les trois catégories d'objection au cours ECR partagent un refus d'adhérer à l'argumentaire étayant les deux finalités du programme, soit la «reconnaissance de l'autre» et la "poursuite du bien commun » ${ }^{1}$. La première, écrit Leroux, renvoie à des considérations relatives à la liberté de conscience et de religion, selon lesquelles «le programme résulterait d'une conception de la sécularisation reposant sur des fondements philosophiques inacceptables, en particulier le relativisme, perçu comme la doctrine fondamentale orientant le programme tant dans le domaine religieux que dans le domaine moral ${ }^{2}$. Cette position est à la fois associée à des parents catholiques militant pour l'exemption de leurs enfants du cours ECR, dont ceux

1. Ministère de l'Education, du Loisir et du Sport (2008). Programme Éthique et culture religieuse. Enseignement secondaire. Québec, gouvernement du Québec, http://www.education. gouv.qc.ca/programme-ethique-et-culture-religieuse/

2. Georges Leroux, Différence et liberté, Montréal, Boréal, 2016, p. 68. 
qui ont plaidé leur cause jusqu'en Cour suprême en $2012^{3}$, et au Collège Loyola de Montréal, qui a obtenu le droit de cette même instance, en 20I 5 , d'enseigner un programme "équivalent " à celui d'ECR, en cohérence avec son éthos catholique 4 . Une deuxième catégorie d'objections, nous dit Leroux, concerne les acteurs dans la mouvance du Mouvement laïque québécois et de ses acolytes, un groupe fermement opposé à toute présence du religieux dans la sphère publique, qui s'oppose à un enseignement culturel des religions à l'école depuis les premiers débats sociaux sur la question en I999. En dernier lieu, Leroux repère les objections relatives "aux conséquences idéologiques et politiques du programme, perçues par certains comme porteur d'un endoctrinement multiculturaliste" et donc, comme un vecteur potentiel de "dénationalisation», selon l'expression de Mathieu Bock-Côté.

Ces trois catégories d'objection incarnent chacune à leur manière, comme le montre bien Leroux, un parti pris contre le pluralisme en éducation, mais en déployant un argumentaire passablement différent. C'est probablement dans le cas de la critique laïciste que l'on retrouve la disqualification la plus visible du religieux en tant que réservoir de "croyances». Dans cette position, dont la filiation remonte directement à la philosophie des Lumières et au paradigme de la sécularisation ${ }^{5}$, la religion est associée à un pendant de la méconnaissance, à une forme d'obscurantisme vouée à une disparition complète dans la modernité avancée. Cette vision "anticléricale " de la laïcité ${ }^{6}$, au fondement de la conception française de la séparation entre l'Église et l'État, est donc porteuse d'un profond mépris pour la religion, perçue comme un reliquat de la tradition, un signe de faiblesse, voire de crédulité et d'ignorance. Sur le plan éducatif et politique, cette conception préconise ainsi un effacement de la religion dans les institutions publiques et les lieux de délibération collective, n'étant pas perçue comme une ressource compatible avec la «raison publique», universelle.

L'objection nationaliste repérée par Leroux se rapproche quant à elle de la laïcité de "foi civique ", définie par Milot ${ }^{7}$, qui tend à mettre en concurrence les valeurs portées par les traditions religieuses et celles de la société politique. À la différence de la critique précédente, le point focal de cet argumentaire ne concerne pas tant le statut épistémologique de la religion que le risque de dissolution ad hoc de la majorité découlant de l'imposition d'une conception relativiste du religieux. Comme le fait remarquer Leroux,

3. Comme le rapporte Leroux, dans la note 5 de la page 66 de son ouvrage, la Cour suprême a rejeté la plainte du couple Lavallée-Jutras de Drummondville au nom des obligations de l'État québécois en matière d'éducation publique.

4. Pour plus de détails, voir la note 6 de la page 67 de l'ouvrage de Georges Leroux.

5. Jean-Paul Willaime, Sociologie des religions, Paris, Presses universitaires de France, I995, p. 89-93.

6. Micheline Milot, La laïcité en 25 questions, Montréal, Novalis, 2008, p. 50-54.

7. Ibid., p. 58-62. 
cette critique n'entretient pas un rapport clair à la religion majoritaire, mais, dans plusieurs cas, celle-ci n'est pas discréditée dans la mesure où elle apparait comme un marqueur objectif de la mémoire collective, chère aux tenants de cette position ${ }^{8}$. Fait intéressant: cette objection est souvent exprimée dans un champ sémantique apparenté à l'imaginaire du complot, qui suggère que l'État québécois aurait tenté d' «endoctriner » les enfants à l'idéologie multiculturaliste. Cette tangente est particulièrement claire dans l'extrait suivant, tiré de l' 'étude » de Joëlle Quérin' sur le programme ECR par Leroux ${ }^{10}$ : "Le programme a été préparé par l'État québécois en vue d'une "transformation radicale de la société québécoise en la reprogrammant à partir du logiciel idéologique du multiculturalisme".» Or, comme le soulignent Bronner ${ }^{11}$ et d'autres ${ }^{12}$, cet argumentaire qui passe rarement au crible de l'examen des faits tend à justifier les idéologies les plus extrêmes, y compris certaines formes de nationalisme identitaire, la xénophobie et le racisme, qui entretiennent à des degrés divers un rapport étroit à l' "imaginaire du complot mondial » censé rendre compte de l'histoire et de la politique.

Enfin, les objections catholiques au programme ECR, en particulier celles exprimées par le couple Lavallée-Jutras, représentent un cas de figure particulier. En effet, les parents de Drummondville souhaitent reconstruire un espace privé encapsulé, selon une sorte d' «utopie pratiquée ${ }^{13}$ ", qui leur permettrait de vivre à l'échelle de la famille une éducation centrée sur ses valeurs catholiques. Paradoxalement, leur position inflexible en faveur d'un opting out de l'éducation libérale au pluralisme ne les empêche pas d'utiliser les outils juridiques du libéralisme politique, la liberté de conscience et de religion, pour revendiquer leur droit à l'exemption.

Malgré leurs différences, ces trois figures de la négation du pluralisme ont en commun une crainte d'effritement du lien social, de l'anomie, dans un contexte où se multiplieraient les "particularismes " religieux et où disparaîtrait tout horizon commun, qu'il soit laïque, national ou existentiel. Certes, le pluralisme a des effets dissolvants sur les croyances collectives, comme le souligne Willaime ${ }^{14}$ :

8. Mathieu Bock-Côté, "Les derniers cathos », Le Journal de Montréal, 2 juillet 2016, [http://www.journaldemontreal.com/2016/07/02/les-derniers-cathos]

9. Joëlle Quérin, Le cours Éthique et culture religieuse. Transmission de connaissances ou endoctrinement? Montréal, Institut de recherche sur le Québec, 2009.

10. Leroux, Différence et liberté, p. I I 2.

11. Gérald Bronner, La démocratie des crédules, Paris, Presses universitaires de France, 20I3.

12. Jérôme Jamin, Imaginaire du complot. Discours d'extrême droite en France et aux États-Unis, Amsterdam, Amsterdam University Press, 2009.

13. Henri Desroche, Communismes religieux, Encyclopaedia Universalis, 20I6, [http:// www.universalis.fr/encyclopedie/communismes-religieux/]

14. Willaime, Sociologie des religions, p. I02. 
couplé à une perte de plausibilité culturelle du religieux, [le pluralisme] accentuera la relativisation de celui-ci: la simple juxtaposition de cultures religieuses diverses au sein d'une même société contribue à relativiser la vérité de chacune d'elles et accentue le processus d'individualisation de la religion.

Ce faisant, il contribue nécessairement à diluer les valeurs substantives religieuses ou nationales — traditionnellement partagées par tous. Cette observation est partagée par Berger ${ }^{15}$ qui ajoute que la modernité tend à pulvériser les "structures intermédiaires ", référant aux lieux de socialisation porteurs de sens pour l'individu, dont la famille, le voisinage et les associations de la société civile, laissant alors les individus désemparés, devant une anomie profonde. Même si elle n'est pas explicite dans les trois objections, cette peur du «vide» et de la rupture du lien social ressort de différentes manières de chacun de ces argumentaires, qui refusent de croire qu'une éducation au pluralisme puisse assurer la "poursuite du bien commun » ou que le cours ECR reconstruise une «structure intermédiaire» valide.

\section{Deux objections catholiques, une vision opposée du pluralisme}

Or, si on se penche plus spécifiquement sur les deux types d'objection catholique au programme ECR, qui ont donné lieu aux deux causes entendues en Cour suprême en 2012 et en 20I 5 , on peut constater qu'elles ne s'articulent pas autour des mêmes enjeux ni ne visent les mêmes finalités. Nous ne considérons donc pas, comme Leroux, qu'elles se répercutent de la même manière sur le fait pluraliste et l'universalité démocratique. Ce dernier écrit en effet que "l'objection communautariste catholique", qui recouvre à la fois la position du couple Lavallée-Jutras et celle du Collège Loyola, renvoie à « un passé idéalisant, qui refuse de prendre en compte les conséquences de la modernité dans des sociétés comme la nôtre. On peut certes se sentir nostalgique de ces communautés homogènes et fortement ritualisées, soumises à une autorité unique, mais qui contestera que ces communautés ne sont pas l'habitat type du Québec d'aujourd'hui ? $^{16}$." Si cette interprétation qualifie précisément, à notre sens, la vision que nous décririons même comme «fondamentaliste» des parents catholiques, elle nous semble décalée par rapport à celle de Loyola, qui ne s'oppose pas aux finalités de l'éducation au pluralisme, mais souhaite l'appuyer sur un "particularisme universel ${ }^{17}$ ».

15. Peter Berger, The Sacred Canopy: Elements of a Sociological Theory of Religion, New York, Anchor Books.

16. Leroux, Différence et liberté, p. 90.

17. Barnaby Riedel, «Universal Particularism: Making an Ethical Islamic School in Chicago ", in M. Minow et al., dir., Just Schools, New York, Russell Sage Foundation, 2008, p. I32-I65. 


\section{Le couple Lavallée-Jutras et le refus «fondamentaliste» de la modernité}

La plainte du couple Lavallée-Jutras, entendue en Cour suprême, allègue que le cours ECR entraîne des préjudices graves sur leur enfant, allant jusqu'à menacer sa liberté de conscience et de religion, dans la mesure où ce programme "aborde le phénomène religieux dans le cadre d'un cours qui prétend à la "neutralité » ", mais qui exposerait en réalité "au courant philosophique mis de l'avant par l'État: le relativisme ${ }^{18}$ ». Cette allégation n'est pas unique au cas du cours ECR ni exclusive au contexte québécois. De nombreuses plaintes de ce genre ont notamment été entendues aux ÉtatsUnis, notamment la populaire cause Mozert vs Hawkins County (I983), dans laquelle un groupe de parents du comté de Hawkins au Tennessee a allégué que le programme de lectures obligatoires prévu au programme scolaire public violait la liberté de conscience et de religion de leurs enfants, au sens où le contenu des lectures proposées (dont The Wizard of $\mathrm{O} z$, Macbeth et The Diary of Anne Frank) exposait les enfants à des visions du monde concurrentes à celles de leurs parents, notamment quant au rôle des femmes. Selon la logique argumentaire déployée par les parents de Hawkins County, l'exposition neutre à différentes visions du monde à travers les lectures prescrites dissimulait un pernicieux mécanisme de reproduction sociale d'une philosophie libérale individualiste. Comme le souligne Stolzenberg dans son analyse de cette cause:

In their eyes, the standpoint of neutrality estranged the children from their parents' tradition by turning religious absolutes into matters of personal opinion. The schools seemingly objective appeal to individual reason plainly inculcated the values of individual choice, toleration and reason, values that rather than transcending culture, derive from and reproduce, liberal, pluralist society ${ }^{19}$.

Ainsi, dans cette logique argumentaire, qui structure aussi le discours des parents de Drummondville, la «neutralité » n'est pas neutre, mais induit une position relativiste dans l'univers du croire, incompatible avec une position croyante "absolue », comme si laisser le choix constituait déjà, en soi, un choix.

Cet argumentaire a fleuri aux États-Unis entre la fin du XIX ${ }^{e}$ siècle et le début du $\mathrm{xx}^{\mathrm{e}}$ siècle, avec la montée du fondamentalisme évangélique, qui critiquait «l'humanisme séculier» (secular humanism), considéré comme un catalyseur de la dilution morale de la société, ferment d'athéisme, favorable à l'enseignement du darwinisme et cherchant à créer un "nouvel ordre

18. S. L. c. Commission scolaire des Chênes, Cour suprême du Canada, 20I 2, p. 25 I. http://scc-csc.lexum.com/scc-csc/scc-csc/fr/item/7992/index.do

19. Nomi Maya Stolzenberg, "He Drew a circle That Shut Me Out": Assimilation, Indoctrination and the Paradox of a Liberal Education, Harvard Law Review, Vol. I06, no. 3, I993, p. 6I2. 
social» socialiste ${ }^{20}$. Forgée comme une catégorie discursive idéologique opposée à celle dans laquelle la religion est souvent socialement située, l'expression a été popularisée entre autres par Schaffer, dans ses ouvrages How Should We Then Live? (1976) et Whatever happened to the Human Race? (1979), et par LaHaye, dans The Battle for the Mind (1980), après avoir surgi en réaction à la publication du manifeste Humanist Manifesto I, par un groupe d'intellectuels américains, en I933. Même si quelques philosophes se sont eux-mêmes définis comme tels (dont l'un des cosignataires du manifeste, John Dewey), l'étiquette d' «humaniste séculier» a principalement été accolée de l'extérieur par les fondamentalistes opposés à toute forme de pensée inscrite dans une vision du monde libérale ou individualiste. Ce mouvement de retour aux sources a coïncidé au début du $\mathrm{xx}^{\mathrm{e}}$ siècle avec une certaine sécularisation interne de l'évangélisme protestant, à la suite de l'importation aux États-Unis de nouvelles méthodes de critiques exégétiques (Higher criticism) ébranlant l'autorité des textes sacrés ${ }^{21}$. À cette crise interne, qui a catalysé l'inerrance biblique (lecture littérale de la Bible) s'est ajouté un ensemble de mutations sociales plus larges, qui ont accentué l'érosion du traditionalisme évangélique, dont l'urbanisation et l'immigration accélérées, l'essor du darwinisme bouleversant la conception traditionnelle de la science (comme explication des Mystères divins), et l'évangélisme social, reléguant à l'arrière-plan certains pans de la métaphysique religieuse ${ }^{22}$. Ainsi, la critique fondamentaliste de l'humanisme séculier récuse à la fois la sécularisation de la religion, en vertu de laquelle le dogme est plus volontiers critiqué, et les valeurs socioculturelles ambiantes, au profit d'un certain conservatisme moral ${ }^{23}$.

Au Québec, les appelants à la Cour suprême ne convoquent pas directement l'expression consacrée d'humanisme séculier, mais définissent plutôt l'idéologie du programme comme "relativiste». Or, comme le précise le juge de la Cour suprême: "Malgré sa sincérité, leur opinion [des parents] selon laquelle un relativisme moral fondamental constitue la caractéristique essentielle de ce programme n'est pas suffisante pour établir une violation de la Charte canadienne ou de la Charte québécoise ${ }^{24}$. Les parents objectent de plus, comme dans la cause américaine précitée, que «l'exposition des enfants à différents faits religieux crée de la confusion chez ces derniers. La confusion ou le "vide" résulterait de la présentation, sur un pied d'égalité, de

20. Moktar Ben Barka, Les nouveaux rédempteurs. Le fondamentalisme protestant aux États-Unis, Genève, Éditions de l'Atelier-Labor et Fides, I998.

21. Ibid. Stolzenberg, "He Drew a circle That Shut Me Out": Assimilation, Indoctrination and the Paradox of a Liberal Education, p. 6I 5.

22. Ben Barka, Les nouveaux rédempteurs. Le fondamentalisme protestant aux ÉtatsUnis, 1998.

23. Olivier Roy, La sainte ignorance. Le temps de la religion sans culture, Paris, Seuil, 2008.

24. S. L. c. Commission scolaire des Chênes, 20I2, p. 259-260. 
croyances différentes ${ }^{25}$.» Ce "vide» fait directement référence, comme dans le cas américain de 1983 , à une trame sociale dépassant largement le cadre scolaire, qui est le fait des sociétés sécularisées et pluralistes, où s'affrontent différentes conceptions de la vie bonne. Mais, comme argue le juge: "l'exposition précoce des enfants à des réalités autres que celles qu'ils vivent dans leur environnement familial immédiat constitue un fait de la vie en société ${ }^{26}$ ».

On voit ainsi clairement, à travers les arguments du couple LavalléeJutras, bien décrits par Leroux, les indicateurs d'une critique «fondamentaliste ", au sens où elle exprime un refus du statut marginal de la religion dans la société et n'accepte pas la confrontation à d'autres visions du monde à travers l'École, nécessairement plurielle dans ce nouveau contexte.

\section{Le Collège Loyola et le catholicisme comme «particularisme universel»}

À la différence de celle du couple de Drummondville, la demande d'exemption au cours ECR du collège Loyola, qui réclamait en réalité le droit d'adapter le contenu du programme à la perspective confessionnelle de son établissement catholique, ne s'oppose pas frontalement aux finalités pluralistes de l'ECR. En fait, la décision de la Cour suprême autorise le Collège, comme il le souhaite, à enseigner la doctrine et les croyances éthiques catholiques du point de vue catholique, tout en exposant les croyances et les normes éthiques d'autres religions «de manière objective et respectueuse ${ }^{27}$ ». De plus, le jugement précise que

les enseignants de Loyola devront s'assurer que le débat a lieu dans un climat de respect, mais, lorsque le contexte de la discussion en classe l'exigera, ils pourront préciser en quoi consistent les convictions catholiques, les raisons pour lesquelles les catholiques y adhèrent et les raisons pour lesquelles d'autres propositions éthiques ou doctrinales sont incompatibles avec ces convictions ${ }^{28}$.

Il ne s'agit donc pas vraiment dans cette requête d'une négation du pluralisme, comme dans la vision précédente, mais davantage d'un "traitement» de la diversité au prisme d'une perspective confessionnelle. Ce type de vision de l'éducation est souvent mis de l'avant, comme je l'ai déjà montré29, dans les écoles confessionnelles du Québec qui cherchent à transmettre certaines valeurs "universelles " grâce au véhicule d'une lignée croyante particulière. En effet, dans les trois écoles juive, musulmane et Steiner que j’ai observées dans la grande région de Montréal, la reproduction d'une orientation confessionnelle (ou spirituelle) ne s'est pas révélée incompatible avec la for-

25. Ibid., p. 254-255.

26. Ibid., p. 237.

27. Loyola c. Québec, Cour suprême du Canada, 20I5, p. 62I, https://scc-csc.lexum. $\mathrm{com} / \mathrm{scc}-\mathrm{csc} / \mathrm{scc}-\mathrm{csc} / \mathrm{fr} / \mathrm{I} 4703 / \mathrm{I} /$ document.do

28. Ibid.

29. Stéphanie Tremblay, Les écoles juive, musulmane et Steiner. Pluralité des voies éducatives, Montréal, Presses de l'Université du Québec, 20 I4. 
mation de citoyens ouverts au pluralisme de la société ambiante. Par exemple, à l'école Steiner, une conception implicite de la citoyenneté s'exprimait à travers la "moralité" vécue en classe, articulée autour de valeurs chrétiennes redéfinies dans leur portée "universelle", telles que l' "amour des autres », le « respect de la nature» et l' «empathie » ${ }^{30}$. De même, à l'école musulmane, les enseignants privilégiaient une double allégeance à l' " oumma » musulmane et à la société québécoise et canadienne, en s'appuyant sur des valeurs présentées comme "musulmanes» (ex. respect des parents et des amis, politesse, sensibilité écologique, etc.), mais laissant voir en même temps une forte adaptabilité de la religion aux contingences sociales. À l'école juive, les discours éducatifs valorisaient à la fois une forte intégration communautaire, en même temps qu'ils insistaient sur une vaste ouverture cognitive et normative à la diversité religieuse, que ce soit par des débats sur l'existence de Dieu, les rapports entre religion et science ou des discussions sur le hijab et d'autres signes religieux. Dans ce cas, l'ouverture était en quelque sorte médiatisée par le judaïsme, qui devenait une voie privilégiée vers la rencontre de l'autre. Ainsi, il semble que, dans certains contextes scolaires,

le vecteur religieux ou spirituel [...] représenterait une sorte de «sésame » pour l'apprentissage de l'ouverture à l'autre. Qu'il s'agisse du christianisme ou de l'anthroposophie, de l'islam ou du judaïsme, ces références comportent une trame commune de valeurs civiques et morales [...] cette connexion à l'absolu semble rendre moins faillibles les valeurs dans lesquelles on croit. Elle postule aussi, plus ou moins implicitement, que le développement de sa propre identité religieuse ou spirituelle constitue un préalable à l'ouverture et à la compréhension de l'autre ${ }^{31}$.

Dans cette vision certes communautarienne de la société, mais pas fondamentaliste en ce qu'elle ne renonce pas à une éducation au pluralisme, on ne voit donc pas de contradiction entre "particularisme » et "universalité ». Il me semble que cette conception du rôle de la religion dans le lien social se rapproche d'ailleurs de l'argument proposé par Leroux sur le pouvoir critique et conceptuel des religions. En se référant aux travaux de Diane L. Moore, l'auteur suggère en effet que les visions religieuses du monde peuvent générer des "cadres conceptuels alternatifs", sur lesquels peut s'appuyer une critique des fondements normatifs implicites de la culture dominante. Dans cette perspective, il suggère que la connaissance des religions peut favoriser «l'imagination culturelle» (cultural imagination) en ce qui concerne l'action humaine et la liberté, en rendant possible «l'examen d'approches, voire de solutions plus justes ou tout simplement plus humaines à des problèmes sociaux complexes ${ }^{32} »$. L'émergence de cette imagination culturelle se

30. Ibid, p. 259.

31. Ibid., p. 26r.

32. Leroux, Différence et liberté, p. 82. 
limite-t-elle nécessairement à une connaissance objective des religions ou bien peut-elle transiter par une posture confessionnelle ouverte à la diversité religieuse? Il semble que la décision de la Cour suprême suggère, au regard du cours ECR, la plausibilité de la deuxième solution.

\section{Conclusion}

Ainsi, si les trois catégories d'objection au cours ECR convergent quant à leur réticence à adopter une conception pluraliste du lien social, faisant d'une certaine manière le deuil d'une culture commune substantive, qu'elle soit nationaliste, laïque ou religieuse, nous avons vu que les critiques catholiques recouvrent deux types d'argumentaires passablement différents. La posture des parents de Drummondville se caractérise en effet par une absence de compromis à l'égard du cours, et plus largement, du fait pluraliste, alors que la position de Loyola accepte d'aller à la rencontre de l'altérité, dans la mesure où cet enseignement passe par un ancrage confessionnel. Indépendamment de la réponse apportée par la Cour à cette requête, l'objection de Loyola, qui ne coupe pas les ponts avec le pluralisme mais propose d'y apporter des ajustements, pose la question des limites de la tolérance libérale. Le modèle d'éducation au pluralisme préconisé au Québec peut-il imposer une présentation neutre et objective des différentes visions du monde, au prix de limiter la tolérance des individus ou des groupes qui s'y opposent, ou bien peut-il s'ajuster à différentes conceptions du pluralisme (entre autres à celle de Loyola) au nom de ses propres principes d'ouverture et de tolérance? La question, délicate, mérite d'être posée. 